\title{
Structural Study on the Cubic to Tetragonal Transformation in Arc-melted $\mathrm{ZrO}_{2}-3$ mol$\% \mathrm{Y}_{2} \mathrm{O}_{3}$
}

\author{
By M. Hayakawa*, M. Tada**, \\ H. Okamoto* and M. Oka*
}

\begin{abstract}
A herringbone structure observed in an as arc-melted specimen was investigated using XRD, TEM and surface relief study. The specimen was cooled on a water cooled copper hearth and the grain size was approximately $1 \mathrm{~mm}$. The results confirmed the crystal structure to be tetragonal $\left(a_{\mathrm{t}}=0.5100\right.$ and $\left.c_{\mathrm{t}}=0.5166 \mathrm{~nm}, c / \mathrm{a}=1.0129\right)$. The orientation relation among the different variants of the tetragonal lattice and their arrangement in the herring bone structure were determined; the structure was shown to be suited for accommodation to the cubic (fluorite structure) to tetragonal transformation strain. Surface relief associated with the transformation was also observed and correlated to the transformation strain. From these results, the transformation was suggested to be of diffusionless type (martensitic).
\end{abstract}

(Received June 19, 1986)

Keywords: zirconia, ceramics, transformation, diffusionless transformation, martensite

\section{Introduction}

Three allotropic phases are known in pure $\mathrm{ZrO}_{2}$, namely the cubic (fluorite type), the tetragonal and the monoclinic structure in a descending order in temperature. The high temperature phases can be partially or fully stabilized by adding an appropriate amount of $\mathrm{Y}_{2} \mathrm{O}_{3}{ }^{(1)}$, or such materials as $\mathrm{CaO}, \mathrm{MgO}$, etc. The tetragonal to monoclinic transformation is well substantiated to be martensitic ${ }^{(2)(3)}$. Toughening of PSZ (partially stabilized zirconia) due to this transformation under the influence of the stress field at a crack tip was first reported by Garvie et $a l .{ }^{(4)}$, and a detailed mechanism of the transformation has been a subject of considerable work $^{(5)-(7)}$. The cubic to tetragonal transformation has also been studied in a plenty of work, and diffusionless transformation was suggested to occur, in addition to the usual diffusional transformation.

Scott $^{(1)}$ was the first to report a "multiply twinned structure" in a specimen rapidly cooled from the melt. He suggested that the struc-

* Department of Mechanical Engineering, Tottori University, Koyama, Tottori 680, Japan.

** Formerly Graduate Student Tottori University, now at Ushio Inc., Tokyo. ture had a tetragonal symmetry and was caused by a displacive transformation from the cubic phase.

Recently Sakuma et al. ${ }^{(8)(9)}$ observed a herringbone structure in an as arc-melted $\mathrm{ZrO}_{2}-3$ mol $\% \mathrm{Y}_{2} \mathrm{O}_{3}$ under TEM. A similar structure was also observed in a sintered $\mathrm{ZrO}_{2}-7 \mathrm{~mol} \%$ $\mathrm{Y}_{2} \mathrm{O}_{3}{ }^{(10)}$. Sakuma et al. ${ }^{(8)(9)}$ suggested that the structure might be rhombohedral because a similar herringbone structure had been found in the $\mathrm{ZrO}_{2}-\mathrm{Sc}_{2} \mathrm{O}_{3}$ system ${ }^{(11)(12)}$, in which the crystal structure was rhombohedral. A rhombic structure was also suggested to metastably exist on a ground surface of sintered yttria PSZ $^{(13)}$.

On the other hand, a herringbone structure similar to that found in as arc-melted $\mathrm{ZrO}_{2}-$ $\mathrm{Y}_{2} \mathrm{O}_{3}$ has been observed in a number of alloys, e.g. In- $\mathrm{Tl}^{(14)(15)}, \mathrm{Mn}-\mathrm{Cu}^{(16)-(18)}, \mathrm{Fe}-\mathrm{Pd}^{(19)}$, etc., when these alloys undergo the fcc to fct transformation. The transformation was verified to be diffusionless and the herringbone structure was well accounted for by the transformation strain accommodation due to twinning. Because of the structural analogy, a similar account may be possible for the transformation of the present ceramics.

In the present study, therefore, the crystal structure of the herringbone structure observed in an as arc-melted $\mathrm{ZrO}_{2}-3 \mathrm{~mol} \% \mathrm{Y}_{2} \mathrm{O}_{3}$ is 
first identified by using XRD and TEM. Then evidence for diffusionless character of the transformation is sought for. This involves an examination of concentration inhomogeneity, observation of surface relief and structural analysis in terms of strain accommodation.

\section{Experimental Procedures}

The starting material was $\mathrm{ZrO}_{2}-3 \mathrm{~mol} \%$ $\mathrm{Y}_{2} \mathrm{O}_{3}$ powder prepared by the coprecipitation method $\left(3.68 \mathrm{Y}_{2} \mathrm{O}_{3}, \quad 0.005 \mathrm{Al}_{2} \mathrm{O}_{3}, 0.140 \mathrm{SiO}_{2}\right.$, $0.003 \mathrm{Fe}_{2} \mathrm{O}_{3}$ and $0.003 \mathrm{Na}_{2} \mathrm{O}$ in weight pct, particle size $23 \mathrm{~nm}$ ). Pellets of $1 \mathrm{~g}$ each were sintered for $2 \mathrm{~h}$ in air at $1673 \mathrm{~K}$. These were then plasma-arc-melted on a water-cooled copper hearth in argon. Since the bottom of a specimen did not melt in the arc, it was necessary to flip the specimen and remelt it. Approximately $600 \mathrm{~s}(10 \mathrm{~min})$ were spent to melt a whole specimen at an arc current of $250 \mathrm{~A}$. After the arc was turned off, the melt was cooled on the hearth; it took usually $30 \mathrm{~s}$ to reach $900 \mathrm{~K}$. Arc-melted specimens were black due to oxygen deficiency, as reported by $\mathrm{Scott}^{(1)}$. A preliminary EPMA study of an as arc-melted specimen showed no detectable compositional segregation.

For metallographic observation, the surface of specimens was subjected to polishing on emery papers to 100 grit, followed by polishing with $1 \mu \mathrm{m}$ diamond paste and a final light polish with $0.3 \mu \mathrm{m}$ alumina paste. The polished surface was etched in $50 \mathrm{HF}-50 \mathrm{HNO}_{3}$ for $2 \mathrm{~h}$.

The crystal structure identification was made by using XRD, both the 2-axis diffractometer method (with $\mathrm{CoK} \alpha$ radiation) and the precession camera method (with MoK $\alpha$ radiation). For the former, arc-melted specimens of several millimeter diameter were halved by a diamond saw and the exposed surfaces were polished with diamond paste so that the layer affected by the cut would be removed. A few such specimens were fixed on a specimen holder with plasticine. Since arc-melted specimens had coarse grains $(\sim 1 \mathrm{~mm})$, the adjustment of the specimen's position in the surface and $\theta$ angle was usually necessary to find a suitably oriented grain to give diffraction peaks. For the precession method, a flake $(\sim 200 \mu \mathrm{m})$ of single crystal was selected from crushed power and oriented by using Laue method.

A detailed structural study of the herringbone structure was made by TEM operated at $200 \mathrm{kV}$. For this purpose, a disk $300 \mu \mathrm{m}$ thick was cut from an arc-melted and annealed $(1473 \mathrm{~K}-3.6 \mathrm{ks})$ specimen. This was polished down to $100 \mu \mathrm{m}$ on emery paper and diamond pasted cloth. The disk was further thinned by ion beams and finally coated with carbon to ensure electron conductivity. On the same specimen, compositional fluctuation associated with the transformed structure was examined by EDAX attached STEM.

Surface relief associated with the transformation was also examined. Since a smooth surface could not be prepared on the high temperature parent phase, this was done on the transformed phase at room temperature by the usual metallographic procedure. Then the specimen was heated by the arc until just the edge of the polished surface started to melt, where the top layer of the polished surface was expected to be the parent cubic phase. After the specimen was cooled, the previously polished surface was examined by interference microscopy (both Nomarski type and He-Ne laser fringe type). In this procedure one would expect no surface relief, if the inverse and the forward transformation proceeded in an exactly reversal way. However, in situ observation of the tetragonal/monoclinic transformation of $\mathrm{ZrO}_{2}$ revealed no marked surface relief upon inverse transformation by heating so that the resulting relief on the cooled specimen was due mainly to the forward transformation ${ }^{(3)}$. The same was expected to be the present case, since the inverse transformation temperature was even higher than the monoclinic to tetragonal transformation temperature in $\mathrm{ZrO}_{2}$.

\section{Results}

\section{Optical microscopy}

A typical optical microstructure of an arcmelted specimen is shown in Fig. 1. The struc- 


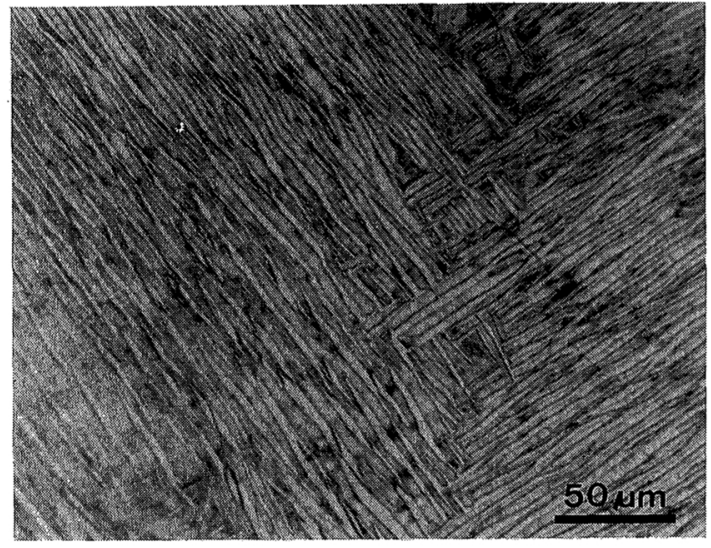

Fig. 1 Optical microstructure of an as arc-melted $\mathrm{ZrO}_{2}-$ $3 \mathrm{~mol} \% \mathrm{Y}_{2} \mathrm{O}_{3}$, etched in $50 \% \mathrm{HF}-50 \% \mathrm{HNO}_{3}$.

ture consists of patches of hundred $\mu \mathrm{m}$ size in which lenticular plates or lamellae are aligned in a direction, presumably a certain crystallographic direction or plane. At a boundary, plates of both directions intertwined to some depths. It is also noted that the interfaces of plates are generally curved. The structure at this magnification did not reveal any herringbone structure in contrast to that in $\mathrm{ZrO}_{2}^{-}$ $\mathrm{Sc}_{2} \mathrm{O}_{3}{ }^{(11)}$.

\section{X-ray diffraction}

The crystal structure of the specimen was identified by XRD. Figure 2 illustrates a diffraction peak profile around (400) peak. Peak positions expected for various lattices are indicated by arrows. It is seen that the observed peaks

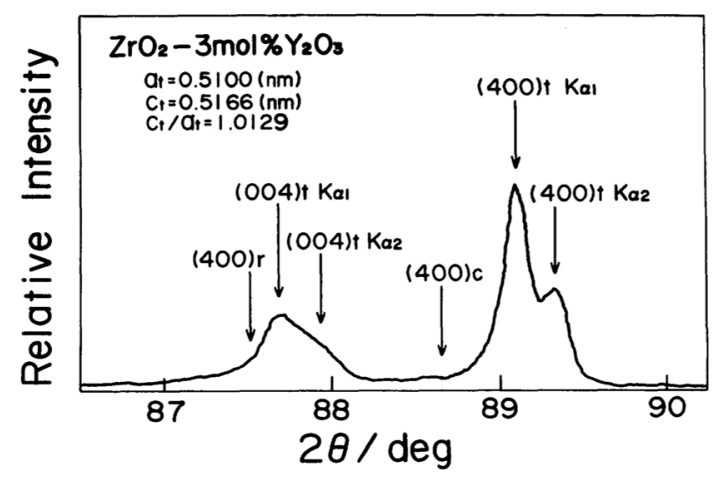

Fig. 2 Diffration peak profile showing only peaks from tetragonal phase. The arrows denote the expected peak positions of the phase indicated. are only those from the tetragonal lattice. The lattice parameters determined from these peaks are $a_{\mathrm{t}}=0.5100$ and $c_{\mathrm{t}}=0.5166 \mathrm{~nm}(c)$ $\mathrm{a}=1.0129$ ). It should be recalled, however, that only a single grain contributed to the profile due to the coarse grained specimen. Therefore, even if other phases exist in the specimen, they would not give rise to any peaks unless the lattice planes are properly oriented with respect to the incident beam. Thus peaks from other phases were searched by rocking the $\theta$ angle by several degrees at the expected $2 \theta$ angles. The range of $\theta$ angle was sufficient, since all the derived lattices are quite close to the parent cubic lattice and consequently the axial and the plane parallelism were expected to be nearly satisfied for all lattices. No peak from other than the tetragonal lattice was found. The simultaneous appearance of (400, $040)_{t}$ and $(004)_{t}$ peaks with an approximate intensity ratio of 2:1 inferred the coexistence of the three variants of the tetragonal lattice in the grain with equal probabilities and the mutual lattice parallelism.

These results were confirmed by precession photographs. Figure 3(a) and (b) are those of the 0 th and the 1 st layer of 100 orientation. Splitting of spots in radial directions due to the tetragonality was observed in high order spots, e.g. 006, and the distance of the split was consistent with the cell dimensions determined from the diffractometer profiles. Again no spots from other than the tetragonal lattice were observed. However, though weak, fluorite-forbidden (112) spot was noted. This was first recognized by Teufer $^{(20)}$ in the high temperature tetragonal phase of $\mathrm{ZrO}_{2}$ and interpreted as arising from the diplacement of oxygen atoms off the planes parallel to the basal plane. The appearance of (112) spot at all $\{112\}_{\text {C }}$ positions $((211)$ spots were verified to appear on the 2nd layer photograph, but not shown here) is another proof of the coexistence of all three tetragonal variants within a crystallite ( $\sim 200 \mu \mathrm{m}$ in size in the present case).

\section{Electron microscopy}

A typical structure observed under the electron microscope-is presented in Fig. 4(a). It exhibits a clear herringbone structure, as 

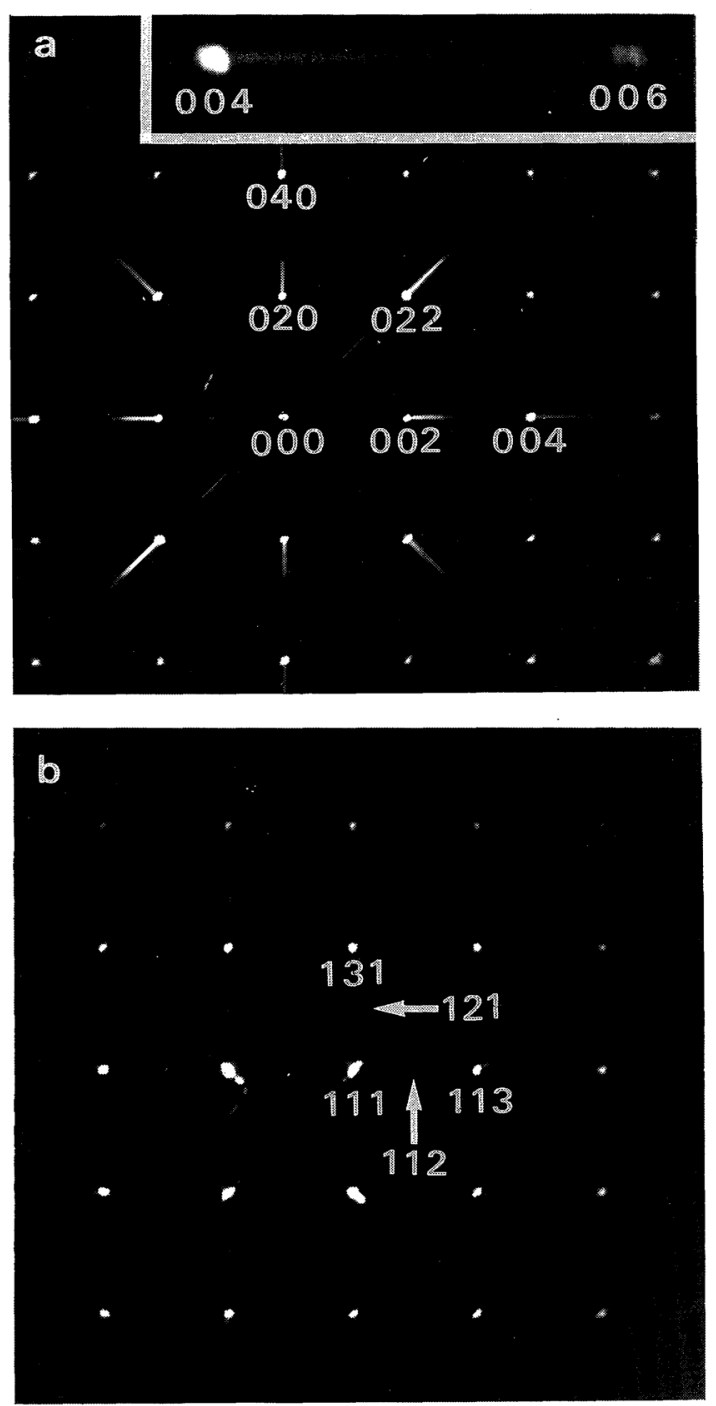

Fig. 3 Precession photographs of (100) orientation. (a) 0 th layer: Splitting of spots due to tetragonality is seen at (040) and (004). (b) 1st layer: Fluorite-forbidden $(112)_{t}$ is seen at (121) and (112) positions.

previously reported by Sakuma et al. ${ }^{(8)(9)}$. The structure consists of alternating bands each of which is characterized by its own orientation of the fault traces. The width of the band is about $1 \mu \mathrm{m}$ and well correlated to the plate thickness in Fig. 1. A diffraction pattern taken from the encircled area in Fig. 4(a) is shown in Fig. 4(b); the indices of the spots and the foil normal are presented in (c). The directions of fault traces $\mathrm{T} 1$ and $\mathrm{T} 3$ and boundary trace $\mathrm{T} 2$ are also included in (c). As illustrated in a stan- dard stereographic projection (Fig. 4(d)), the loci of the three trace normals pass through 110 type poles. Several other micrographs were similarly analyzed and the loci invariably passed near 110 type poles. Thus both the fault planes and the boundary were determined to be $\{110\}$.

In the herringbone structure, a fine tweed like pattern was often observed as revealed in some areas in Fig. 4(a) where the contrast is appropriate. Such a fine structure has been previously reported ${ }^{(8)(9)(21)(22)}$. We shall come back to this point in the last section.

Figure 5 is another electron micrograph of the same foil but tilted so that the incident beam may be $[0 \overline{1} 0]_{\mathrm{t}}$ direction. In this foil direction the (101) $\mathrm{t}$ fault plane in the left band (marked by $b$ ) is perpendicular to the plane of figure, whereas the $(011)_{t}$ fault plane in the right band and $(110)_{t}$ boundary plane are inclined $45^{\circ}$ to the plane of figure. A diffraction pattern taken from area $b$ and the corresponding key diagram are shown in Fig. 5(b) and (c), re= spectively. Splitting of spots normal to the fault plane is clearly seen as illustrated in the inset of Fig. 5(c). Thus the faults are compatible with $(101)_{t}$ twins of $x$ and $z$ variants, where $x$ variant denotes the tetragonal variant whose $c$ axis (long axis) is parallel to the $\mathrm{x}$ axis of the parent cubic lattice; $y$ and $z$ variants are similarly defined. The $c /$ a ratio calculated from the split of (804) $)_{t}$ and (048) $t$ was 1.015, which is in reasonable agreement with the previous XRD result.

When the adjacent band was included as indicated by $d$ in Fig. 5(a), triply split spots were observed; the new spots were successfully indexed as arising from y variant (see the inset in Fig. 5(e)). This is expected, because the right band must be consisting of $\mathrm{y}$ and $\mathrm{z}$ variants since the twin plane is either $(011)_{t}$ or $(0 \overline{1} 1)_{t}$.

In these electron micrographs, each layer of twin is too thin to be identified with the variant by means of selected area diffraction technique. The variant identification is, however, possible from an a priori knowledge of equal probability of all three variants. Namely the thinner plates in both bands must be common to both bands. Together with the knowledge of the twin planes, the layers can be uniquely iden- 

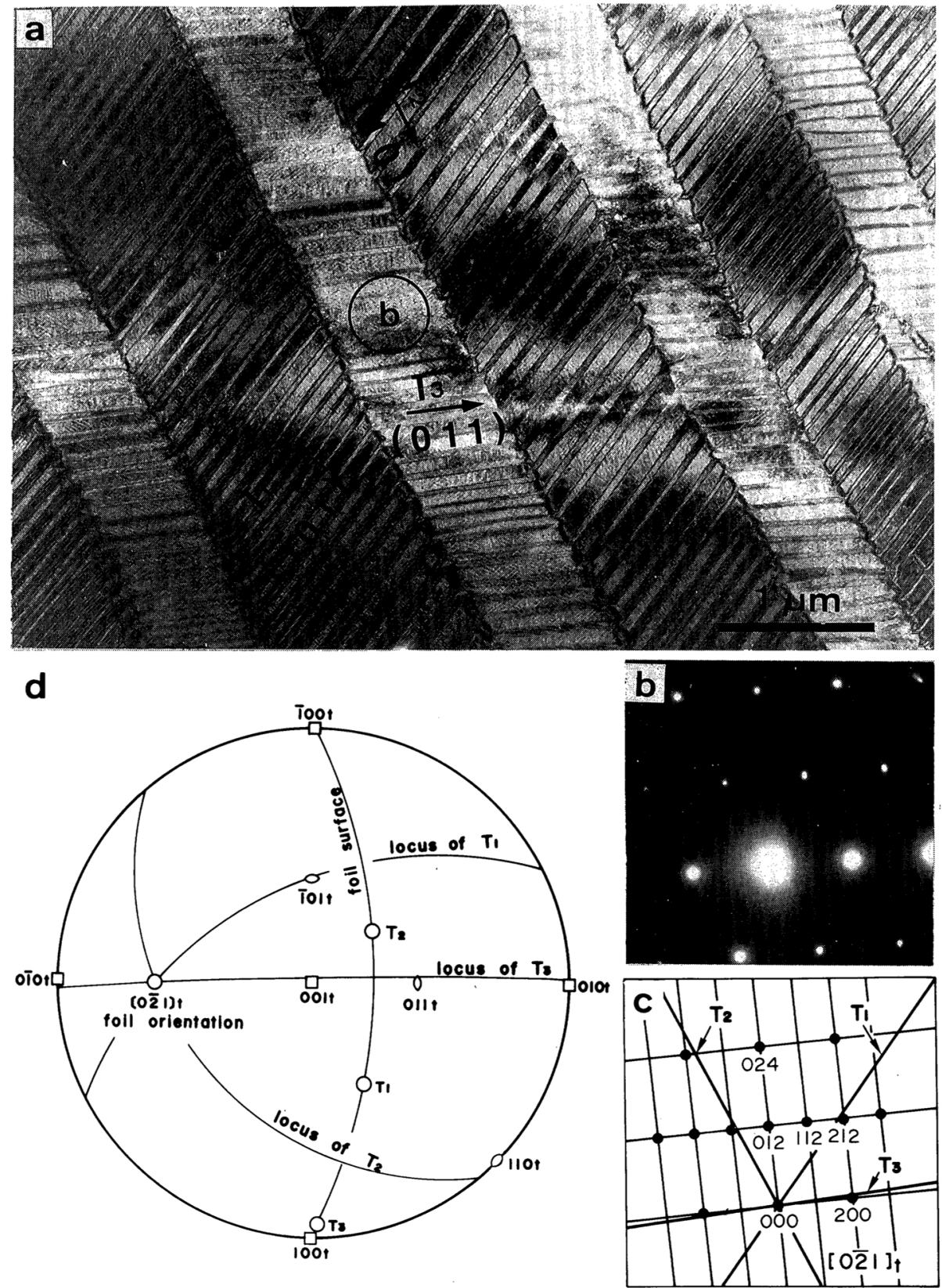

Fig. 4 (a) Electron micrograph of herringbone structure. (b) Diffraction pattern taken from the encircled area marked by $b$ in (a). (c) Key diagram of the diffraction pattern and the fault traces $\mathrm{T} 1$ and T3 and the boundary trace T2. (d) Stereographic projections of the loci of the trace normals. 

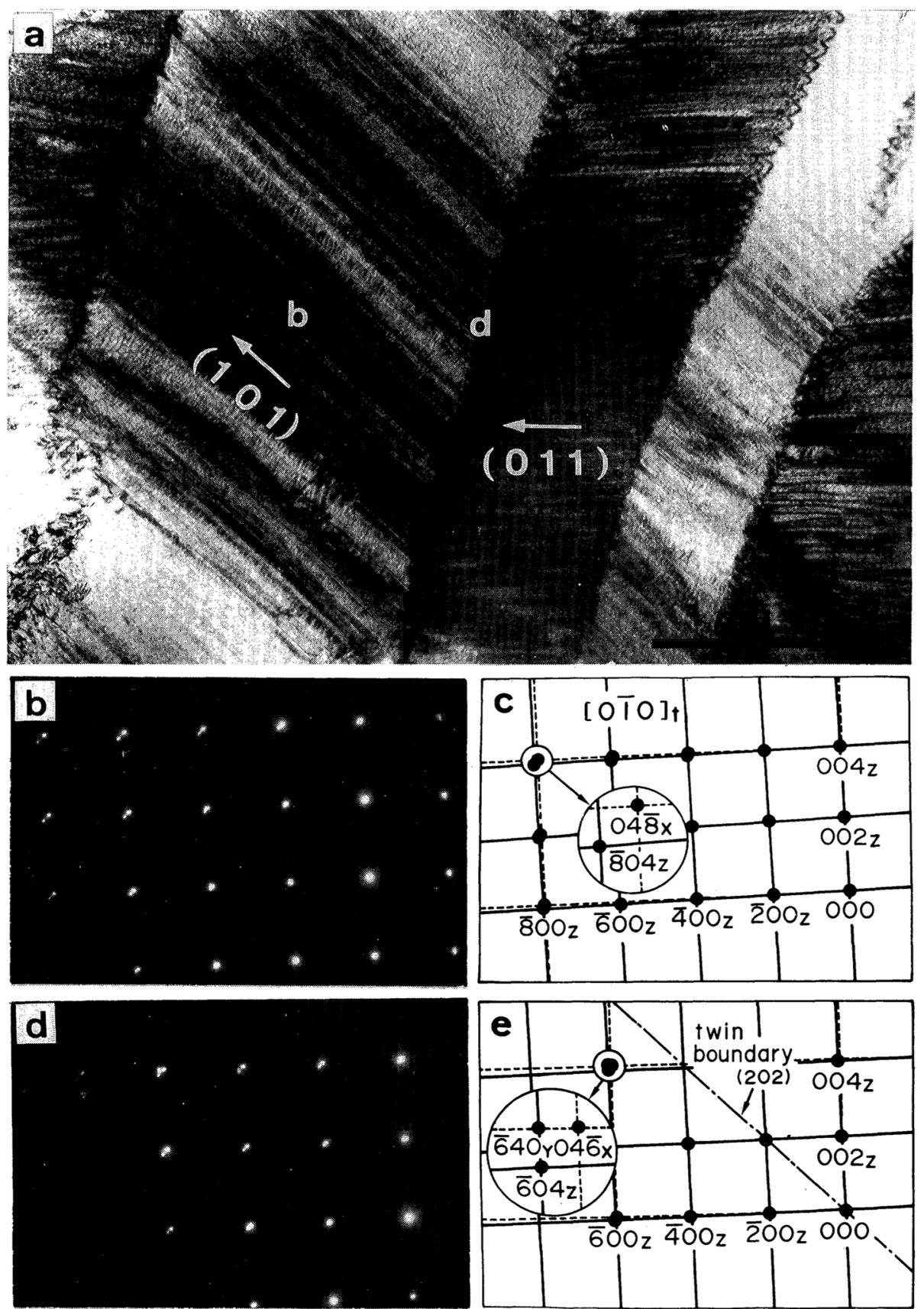

Fig. 5 (a) Electron micrograph with (010) $)_{t}$ incident beam. (b) and (c) Diffraction pattern taken from the encircled area marked by $b$ in (a), and the corresponding key diagram. (d) and (e) same as before but for the encircled area marked by $d$ in (a). 


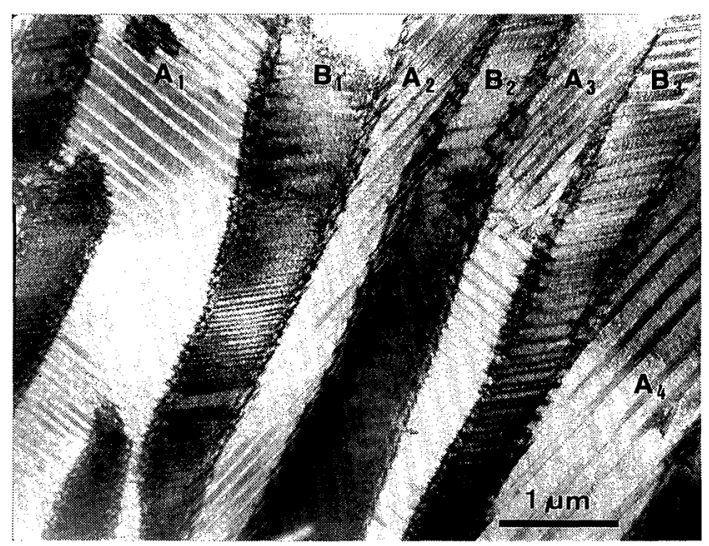

Fig. 6 Herringbone structure where two different (101) twin planes are observed in the same type of bands A1, A2 and A3.

tified with the variants.

The herringbone structures shown above are typical ones, where each of the alternating bands contains a singly oriented twin plane, but in some area two differently oriented twin planes were observed in the same type of bands $A_{1}$ and $A_{2}$ bands) or even in the same band $\left(A_{3}\right.$ band in Fig. 6). Both planes were identified to be of (101) type and perpendicular to each other. This is also expected, because for each combination of the tetragonal variants, there are two equivalent twin planes, e.g. for the $\mathrm{x}-\mathrm{z}$ variant twin $(101)_{t}$ and $(101)_{t}$ are the possible twin planes.

Compositional fluctuation in the herringbone structure was also examined by EDAX attached STEM. A line analysis of yttrium showed no appreciable fluctuation associated with the banded structure (Fig. 7). The result does not, however, rule out the possibility of compositional fluctuation in a finer scale. The absence of appreciable segregation is equally deduced from the sharpness of the XRD peaks in Fig. 2.

\section{Surface relief}

A flat smooth surface was prepared on an as arc-melted specimen. This was then reheated and kept for a few minutes just below the melting point. The surface of the cooled specimen was examined under the interference microscope. In some areas of the surface, upheavals of lenticular shape aligned in definite crystallographic directions were seen (Fig. 8(a)). In other areas, wrinkled upheavals
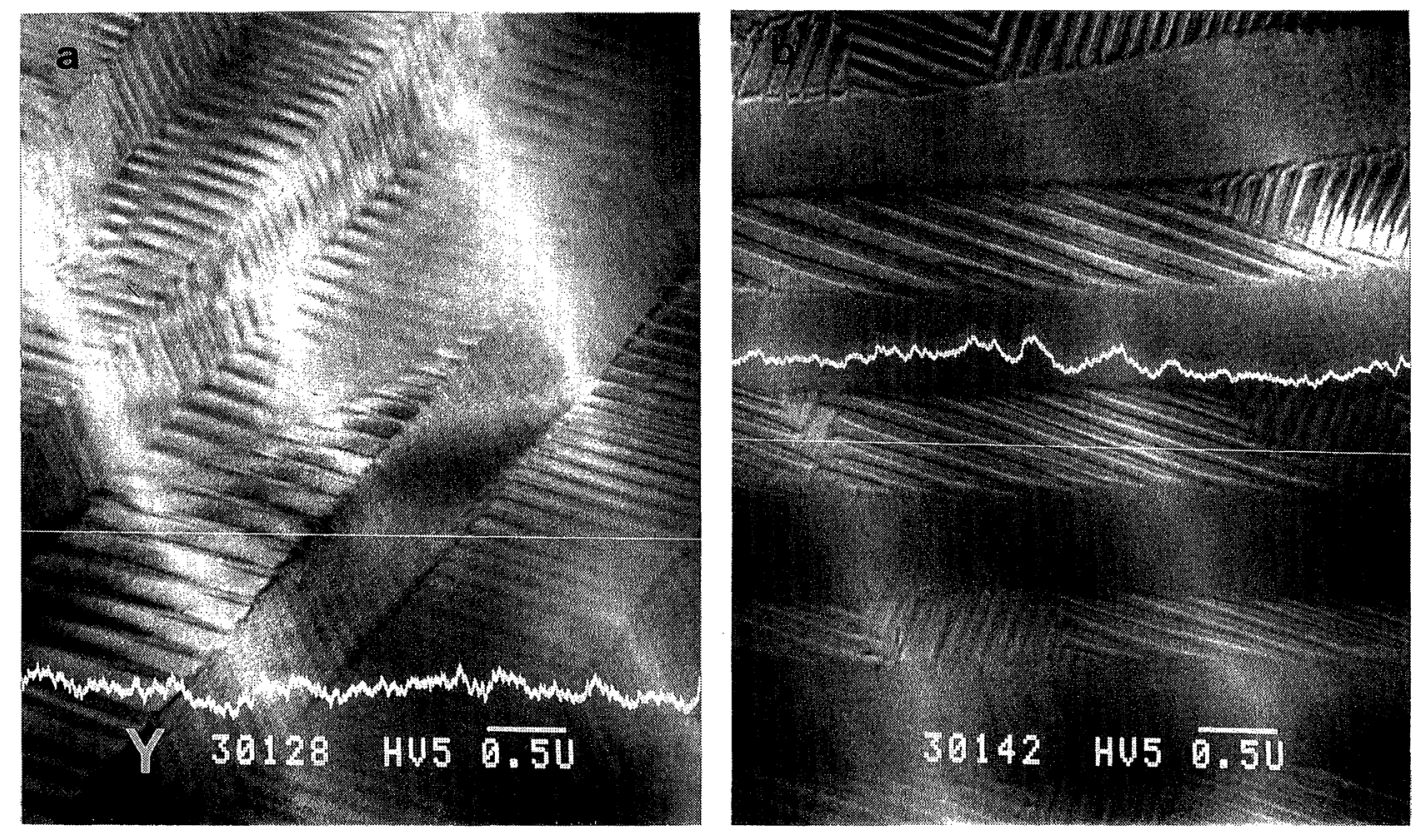

Fig. 7 Line analysis of yttrium composition indicating no perceivable fluctuation associated with the banded structure. 

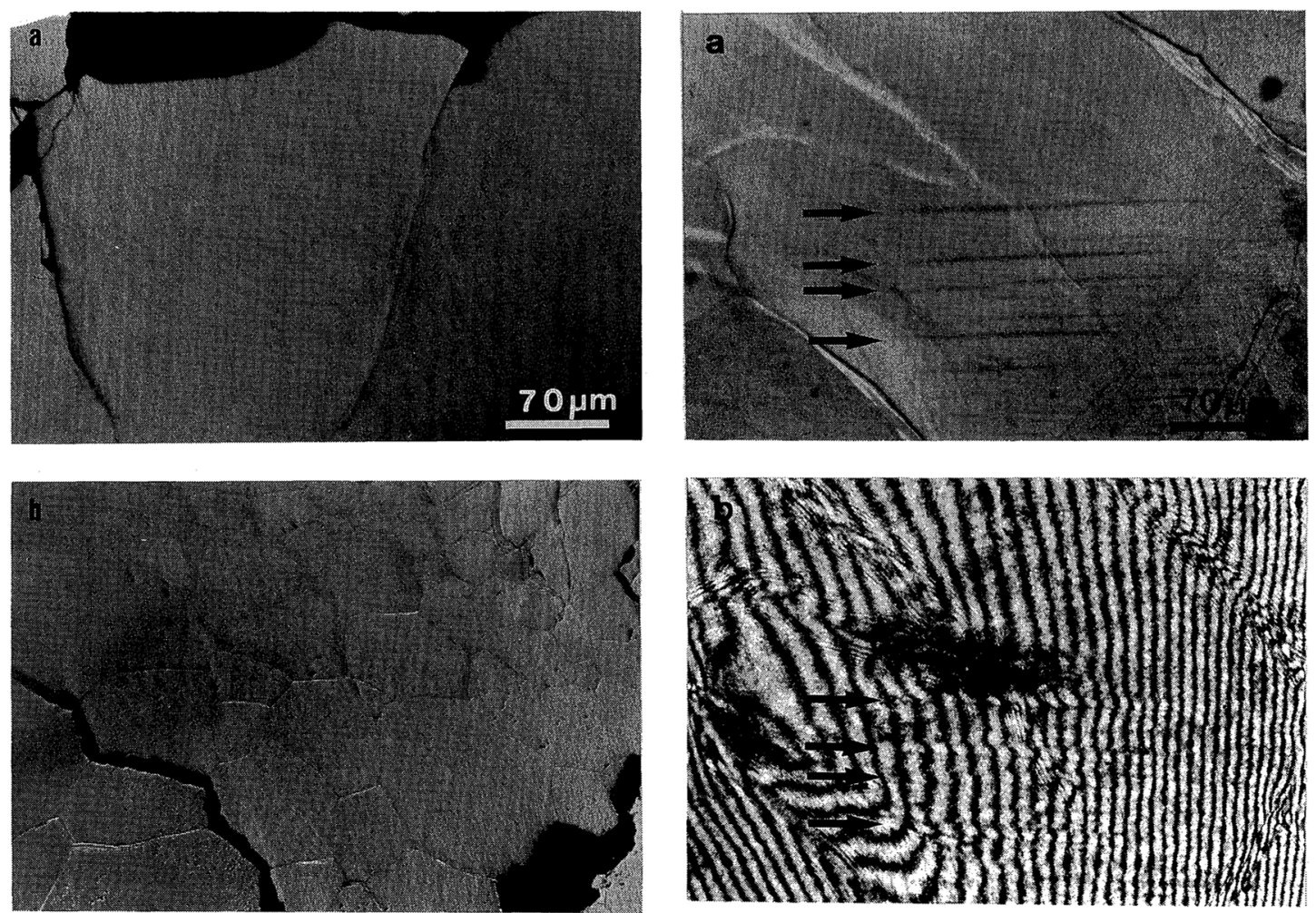

Fig. 8 Two types of surface relief observed in Nomarski interference mode (colored in the originals). (a) Lenticular upheavals. (b) Wrinkled upheavals.

Fig. 9 Surface relief due to large lenticular plates. (a) Nomarski interference mode (colored in the original). (b) $\mathrm{He}-\mathrm{Ne}$ laser interference fringe mode.

were seen (Fig. 8(b)). These photographs were originally taken in the Nomarski mode microscope and revealed clear color contrast; unfortunately much of the contrast was lost in the black and white prints. The former type of upheavals can be readily correlated with the lenticular plates seen in Fig. 1, whereas for the latter the correlation is not obvious. Both types of relief can be considered to be associated with the cubic to tetragonal transformation, since a similar experiment using a fully stabilized $\mathrm{ZrO}_{2}-8 \mathrm{~mol} \% \mathrm{Y}_{2} \mathrm{O}_{3}$ did not exhibit either type of relief. Even for the better defined upheavals of lenticular shape, they were generally too small for one to make a quantitative measurement. Exceptionally large ones are presented in Fig. 9(a) and (b), and these were subjected to the analysis. From the angle of the bent at the boundary $\left(30^{\circ}\right)$ and the wavelength of $\mathrm{He}-\mathrm{Ne}$ laser $(\lambda=633 \mathrm{~nm})$ the tilt angle between the adjacent plates was esti-

mated to be $1-2^{\circ}$. It will be shown in the next section that the value is in reasonable agreement with the strain accommodation model of the herringbone structure.

\section{Discussion}

The present experimental results have clearly revealed that the crystal structure of the herringbone structure observed in an as arc-melted $\mathrm{ZrO}_{2}-3 \mathrm{~mol} \% \mathrm{Y}_{3} \mathrm{O}_{3}$ is tetragonal; the structure is the same as the one reported by Teufer in pure $\mathrm{ZrO}_{2}$ at high temperature ${ }^{(20)}$. The results also clarified how the three tetragonal variants are arranged in the herringbone structure. Some evidence for diffusionless character of the transformation was also pointed out. A quite similar structure has been observed in some alloy systems ${ }^{(14)-(19)}$. In those alloys, the transformation temperature is lower, and better controlled experiments were possible. Such 
experiments verified that the herringbone structure was indeed compatible with a transformation strain accommodation model. The present system is now analyzed in a similar manner.

Let the lattice parameters of the cubic and the tetragonal lattice be $a_{\mathrm{c}}, a_{\mathrm{t}}$, and $c_{\mathrm{t}}$. Then the transformation strain tensor from cubic to $\mathrm{x}$ variant of tetragonal lattice (lattice parallelism is assumed) may be expressed as:

$$
\varepsilon_{x}=\left(\begin{array}{ccc}
\varepsilon_{3} & & \\
& \varepsilon_{1} & \\
& & \varepsilon_{1}
\end{array}\right),
$$

where $\varepsilon_{1}=a_{\mathrm{t}} / a_{\mathrm{c}}-1$ and $\varepsilon_{3}=c_{\mathrm{t}} / a_{\mathrm{c}}-1$. Similarly, the strain tensors for $\mathrm{y}$ and $\mathrm{z}$ variants are

$$
\varepsilon_{y}=\left(\begin{array}{ccc}
\varepsilon_{1} & & \\
& \varepsilon_{3} & \\
& & \varepsilon_{1}
\end{array}\right)
$$

and

$$
\varepsilon_{z}=\left(\begin{array}{lll}
\varepsilon_{1} & & \\
& \varepsilon_{1} & \\
& & \varepsilon_{3}
\end{array}\right),
$$

respectively.

A herringbone structure is schematically illustrated in Fig. 10. The band thickness is set equal, and the thickness of the common variant, i.e. $z$ variant in the present case, is set half the thickness of the other two variants so that the equal probability of the variants is fulfilled as a whole. The latter condition was also experimentally verified by measuring the variant thickness along a few bands.

The relative strain of $\mathrm{x}$ variant with respect to $\mathrm{z}$ variant in $\mathrm{A}$ band is given by

$$
\varepsilon_{x}-\varepsilon_{z}=\left(\begin{array}{ccc}
\varepsilon_{3}-\varepsilon_{1} & & \\
& 0 & \\
& & \varepsilon_{1}-\varepsilon_{3}
\end{array}\right) .
$$

The matrix represents a pure shear on either $(101)_{\mathrm{t}}$ or $(\overline{101})_{\mathrm{t}}$ plane (see e.g. ref. (23)). Such strain can be visualized as twinning of $\mathrm{z}$ variant on either $(101)_{t}$ or $(101)_{t}$ plane to result in $x$ variant. In Fig. 10, (101) twin plane has been chosen. A similar argument holds for the relative strain of $\mathrm{y}$ variant with respect to $\mathrm{z}$ in $\mathrm{B}$

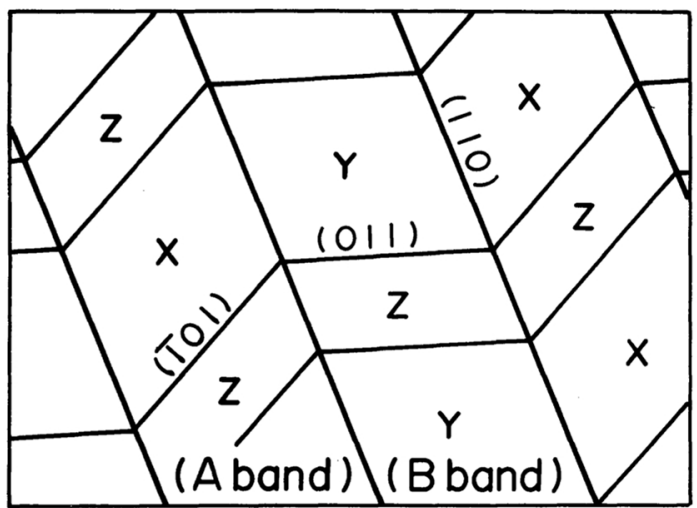

Fig. 10 Schematic representation of an idealized herringbone structure. $\mathrm{x}, \mathrm{y}$ and $\mathrm{z}$ denote the three tetragonal variants (see text for definition).

band. Thus

$$
\varepsilon_{y}-\varepsilon_{z}=\left(\begin{array}{lll}
0 & & \\
& \varepsilon_{3}-\varepsilon_{1} & \\
& & \varepsilon_{1}-\varepsilon_{3}
\end{array}\right),
$$

which is the same as twinning of $z$ variant on either $(011)_{t}$ or $(0 \overline{1} 1)_{t}$ plane to result in $y$ variant. The $(011)_{t}$ plane has been selected here.

Next, the band interface is considered. The average strain of $\mathrm{A}$ band with respect to the matrix is given by

$$
\begin{aligned}
\varepsilon_{A} & =\frac{2}{3} \varepsilon_{x}+\frac{1}{3} \varepsilon_{z} \\
& =\left(\begin{array}{lll}
\left(2 \varepsilon_{3}+\varepsilon_{1}\right) / 3 & & \\
& \varepsilon_{1} & \\
& & \left(2 \varepsilon_{1}+\varepsilon_{3}\right) / 3
\end{array}\right) .
\end{aligned}
$$

Similarly for B band

$$
\varepsilon_{B}=\frac{2}{3} \varepsilon_{y}+\frac{1}{3} \varepsilon_{z}
$$

$=\left(\begin{array}{ccc}\varepsilon_{1} & & \\ & \left(2 \varepsilon_{3}+\varepsilon_{1}\right) / 3 & \\ & & \left(2 \varepsilon_{1}+\varepsilon_{3}\right) / 3\end{array}\right)$.

Thus the relative strain of $\mathrm{B}$ band with respect to $\mathrm{A}$ band is

$$
\varepsilon_{B A}=\varepsilon_{B}-\varepsilon_{A}
$$




$$
=\left(\begin{array}{ccc}
2\left(\varepsilon_{1}-\varepsilon_{3}\right) / 3 & & \\
& 2\left(\varepsilon_{3}-\varepsilon_{1}\right) / 3 & \\
& & 0
\end{array}\right) .
$$

This is again a pure shear on either $(110)_{t}$ or $(\overline{1} 10)_{\mathrm{t}}$ plane. Therefore, it is seen that the band interface is kept macroscopically stress free.

The average strain of the herringbone structure as a whole is given by

$$
\begin{aligned}
\varepsilon_{A v e} & =\left(\varepsilon_{B}+\varepsilon_{A}\right) / 2 \\
& =\frac{2}{3}\left(\begin{array}{lll}
2 \varepsilon_{1}+\varepsilon_{3} & & \\
& 2 \varepsilon_{1}+\varepsilon_{3} & \\
& & 2 \varepsilon_{1}+\varepsilon_{3}
\end{array}\right) .
\end{aligned}
$$

The matrix indicates that the average strain is pure dilatation, as expected. Furthermore, since in the present system the dilatation is approximately zero $^{(21)}$,

$$
2 \varepsilon_{1}+\varepsilon_{3}=0 \text {. }
$$

These calculations show that the herring bone structure is very suited for the transformation strain accommodation. Consequently, the herringbone structure can be regarded as a consequence of the accommodation to the strain due to diffusionless transformation.

Finally the tilt angle between the alternating bands of the present model is calculated and compared with the experimental observation. Referring to eq. (4) the tilt or shear angle between $\mathrm{A}$ and $\mathrm{B}$ bands may be expressed by

$$
\tan \gamma=\frac{4}{3}\left(\varepsilon_{3}-\varepsilon_{1}\right)
$$

The observed lattice parameters and eq. (6) yield $\varepsilon_{1}=-0.0043$ and $\varepsilon_{3}=0.0086$. Substitution of these values for eq. (7) results in $\gamma=1^{\circ}$. This is the maximum observable tilt angle of the surface, where the axis of tilt lies on the surface. When the axis of tilt is inclined to the specimens surface, the observed tilt of the surface is smaller. On the other hand, when the relative fraction of the tetragonal variants or the band thickness deviates from the ideal structure, the surface tilt may become larger. Considering these uncertainties, one may regard the observed value of $1-2^{\circ}$ as in reasonable agreement.
All the experimental results except for the tweed structure have been consistently interpreted within the presently proposed regime of diffusionless transformation from cubic to tetragonal phase. The tweed structure is very similar to those previously observed ${ }^{(8)(10)(21)(22)}$. The contrast was interpreted as arising from either fine precipitates of the tetragonal phase in the cubic matrix ${ }^{(10)(21)}$ or spinodal decomposition toward the tetragonal and the cubic phase $^{(8)(22)}$.

Since the tweed structure is often observed in the absence of the herringbone structure and in a more or less aged specimen, it is considered not to be inherent in the herringbone structure. Nevertheless, it is important in the presently proposed model when the tweed structure develops, since if it develops prior to the herringbone structure the parent phase will be no longer the ideal cubic lattice. Even if this is the case, however, the phase separation in the tweed structure must not be extensive, as all the diffraction results (XRD and TEM) were successfully interpreted disregarding such phase separation. The parent phase with such minor phase separation in a fine scale $(<20$ $\mathrm{nm})$ can be approximated as cubic when averaged over a larger scale, say a size of a single variant of the herringbone structure. Therefore, the strain accommodation mechanism is still operative, provided that the energetic requirement is fulfilld.

On the contrary, if the tweed structure develops after the formation of the herringbone structure, the present model is applicable as it is. But now the tweed structure must be interpreted as the phase separation from solute rich tetragonal to solute poor tetragonal and solute rich cubic. such phase separation has not been reported in this system, but it is certainly possible in view of the equilibrium phase diagram. Further work is required to clarify the nature of the tweed structure in the herringbone structure.

\section{Summary}

Crystallographic investigations were made on the herringbone structure observed in an as arc-melted $\mathrm{ZrO}_{2}-3 \mathrm{~mol} \% \mathrm{Y}_{2} \mathrm{O}_{3}$ specimen. The 
specimen was cooled on a water cooled copper hearth and the grain size was $\sim 1 \mathrm{~mm}$. The results are as follows:

(1) The crystal structure is tetragonal with parameters $a_{\mathrm{t}}=0.5100$ and $c_{\mathrm{t}}=0.5166 \mathrm{~nm}, c /$ $\mathrm{a}=1.0129$.

(2) The structure consists of alternation of two types of bands bounded by (110)t planes; one consists of stacks of (101) twinned layers, the other $(011)_{t}$ twinned layers. Such a structure was shown to be suited for accommodation to the transformation strains.

(3) The transformation from the cubic to this tetragonal herringbone structure is accompanid by surface relief, which is consistent with the transformation strain.

From these results, it was concluded that the herringbone structure is a product of diffusionless transformation from the cubic phase.

\section{Acknowledgements}

The authors wish to thank Toyo Soda Manufacturing Co. Ltd. for supplying the powder materials, Dr. H. Kubo at Nippon Steel Corporation for ion beam thinning the specimens, Drs. K. Kiyonaga and H. Sezaki at Hitachi Metals Co. for STEM analysis, Professor T. Tsukihara at Tottori University for letting us use a precession camera. They also thank Professor H. Iwahara at Tottori University and Professor T. Sakuma at Tohoku University for valuable discussion.

\section{REFERENCES}

(1) H. G. Scott: J. Mat. Sci., 10 (1975), 1527.

(2) G. M. Wolten: J. Amer. Ceram. Soc., 46 (1964), 418.
(3) G. K. Bansal and A. H. Heuer: Acta Met., 20 (1972), 1281; ibid., 22 (1974), 409.

(4) R. C. Garvie, R. H. Hannink and R. T. Pascoe: Nature, 258 (1975), 703.

(5) D. L. Porter, A. G. Evans and A. H. Heuer: Acta Met., 27 (1979), 1654.

(6) A. G. Evans, N. Burlingame, M. Drory and W. M. Kriven: Acta Met., 29 (1981), 447.

(7) F. F. Lange: J. Mat. Sci., 17 (1982), 225; ibid., 17 (1982), 235.

(8) T. Sakuma, T. Yoshizawa and H. Suto: J. Mater. Sci., 20 (1985), 1085.

(9) T. Sakuma, T. Yoshizawa and H. Suto: J. Mater. Sci., 20 (1985), 2399.

(10) A. H. Heuer and M. Rühle: Advances in Ceramics, Vol. 12, Science and Technology of Zirconia II The American Ceramic Society, (1984) p. 1; R. Chaim, M. Rühle and A. H. Heuer: J. Amer. Ceram. Soc., 68 (1985), 427.

(11) R. Ruh, H. J. Garrett, R. F. Domagala and V. A. Patel: J. Amer. Ceram. Soc., 60 (1977), 399.

(12) F. K. Moghadam, T. Yamashita, R. Sinclair and D. A. Stevenson: J. Amer. Ceram. Soc. 66 (1983) 213.

(13) H. Hasegawa: J. Mat. Sci. Letter, 2 (1983) 91.

(14) J. S. Bowles, C. S. Barret and L. Guttman: J. Metals, 188 (1950), 1478.

(15) M. W. Burkart and T. A. Read: J. Metals, (1953), 1516.

(16) Z. S. Basinski and J. W. Christian: J. Inst. Metals, 80 (1952), 659.

(17) E. T. Worrell: J. Appl. Phys., 19 (1948), 929.

(18) O. Nittono, T. Satoh and Y. Koyama: Trans. JIM, 22 (1981), 225

(19) R. Oshima: Scripta Met., 15 (1981), 829; M. Sugiyama, R. Ohsima and F. E. Fujita: J. Japan Inst. Metals, 48 (1984), 881 (in Japanese).

(20) G. Teufer: Acta Cryst., 15 (1962), 1187.

(21) R. H. J. Hannink: J. Mater. Sci., 13 (1978), 2487.

(22) T. Sakuma, Y. Yoshizawa and H. Suto: J. Mater. Sci., 21 (1986), 1436.

(23) J. F. Nye: Physical Properties of Crystals, Oxford (1957) Chapt. 6. 